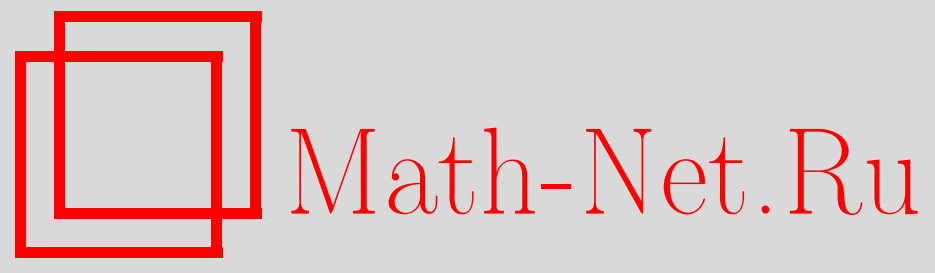

P. М. Сафина, Задача Дирихле для уравнения смешанного типа с сильным характеристическим вырождением и сингулярным коэффициентом, Вестн. Сам. гос. техн. ун-та. Сер. Физ.мат. науки, 2017, номер 1, 80-93

DOI: https://doi.org/10.14498/vsgtu1495

Использование Общероссийского математического портала MathNet.Ru подразумевает, что вы прочитали и согласны с пользовательским соглашением

http: //www . mathnet.ru/rus/agreement

Параметры загрузки:

IP : 54.164 .48 .24

26 апреля 2023 г., 18:29:56

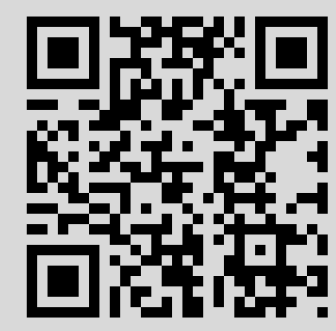


Вестн. Сам. гос. техн. ун-та. Сер. Физ.-мат. науки. 2017. Т. 21, № 1 . С. $80-93$ ISSN: 2310-7081 (online), 1991-8615 (print)

УДК 517.956.6

\title{
Задача Дирихле для уравнения смешанного типа с сильным характеристическим вырождением и сингулярным коэффициентом
}



\section{P. М. Сафина}

Поволжская государственная академия физической культуры, спорта и туризма, Россия, 420010, Казань, Деревня Универсиады, 35.

\begin{abstract}
Аннотация
Для уравнения смешанного типа второго рода с сингулярным коэффициентом исследована первая граничная задача в прямоугольной области. Для исследования поставленной задачи используются методы спектрального анализа. Предварительно решается одномерная спектральная задача. Установлен критерий единственности решения задачи. Единственность решения задачи доказывается на основании полноты системы собственных функций соответствующей одномерной спектральной задачи. Решение задачи построено в явном виде как сумма ряда Фурье-Бесселя. При обосновании равномерной сходимости построенного ряда возникает проблема малых знаменателей. В связи с этим найдена оценка отделенности от нуля малого знаменателя с соответствующей асимптотикой. Полученная оценка позволила доказать сходимость ряда и его производных до второго порядка включительно, а также теорему существования в классе регулярных решений данного уравнения.
\end{abstract}

Ключевые слова: уравнение смешанного типа, задача Дирихле, сингулярный коэффициент, спектральный метод, единственность, ряд Фурье-Бесселя, малые знаменатели, существование.

Получение: 3 июня 2016 г. / Исправление: 17 января 2017 г. / Принятие: 13 марта 2017 г. / Публикация онлайн: 27 апреля 2017 г.

\section{Научная статья}

(2) (7) Контент публикуется на условиях лицензии Creative Commons Attribution 4.0 International (https://creativecommons.org/licenses/by/4.0/deed.ru)

\section{Образец для цитирования}

Сафина Р. М. Задача Дирихле для уравнения смешанного типа с сильным характеристическим вырождением и сингулярным коэффициентом // Вестн. Сам. гос. техн. ун-та. Сер. Физ.-мат. науки, 2017. Т. 21, № 1. С. 80-93. doi: 10.14498/vsgtu1495.

Сведения об авторе

Римма Марселевна Сафина (D) http://orcid.org/0000-0002-5787-1427

старший преподаватель; каф. физико-математических дисциплин и информационных технологий; e-mail:rimma77705@mail.ru 
1. Постановка задачи. Рассмотрим уравнение смешанного типа второго рода с сингулярным коэффициентом

$$
L u \equiv u_{x x}+(\operatorname{sgn} y)|y|^{m} u_{y y}+\frac{k}{x} u_{x}-a^{2} u=0
$$

в прямоугольной области

$$
D=\{(x, y) \mid 0<x<l,-\alpha<y<\beta\}
$$

где $k<1, k \neq 0, l, a \geqslant 0,1 \leqslant m<2, \alpha>0, \beta>0$ - заданные действительные числа.

ЗАДАЧА ДиРИхЛЕ. Найти функиию $u(x, y)$, удовлетворяющую следующим условИЯм:

$$
\begin{gathered}
u(x, y) \in C^{2}\left(D^{+} \cup D^{-}\right) \cap C(\bar{D}) \\
\lim _{y \rightarrow 0+0} y^{m-1} u_{y}(x, y)=-\lim _{y \rightarrow 0-0}(-y)^{m-1} u_{y}(x, y), \quad 0<x<l, 1<m<2, \\
\lim _{y \rightarrow 0+0} \frac{u_{y}(x, y)}{\ln y}=-\lim _{y \rightarrow 0-0} \frac{u_{y}(x, y)}{\ln (-y)}, \quad 0<x<l, m=1, \\
L u(x, y) \equiv 0, \quad(x, y) \in D^{+} \cup D^{-}, \\
u(x, \beta)=\varphi(x), \quad u(x,-\alpha)=\psi(x), \quad 0 \leqslant x \leqslant l, \\
u(l, y)=0, \quad-\alpha \leqslant y \leqslant \beta, \\
u(0, y)=0, \quad-\alpha \leqslant y \leqslant \beta
\end{gathered}
$$

где $\varphi(x)$ и $\psi(x)$ - заданные достаточно гладкие функиии,

$$
\begin{gathered}
\varphi(0)=\varphi(l)=\psi(0)=\psi(l)=0, \\
D^{+}=D \cap\{y>0\}, \quad D^{-}=D \cap\{y<0\} .
\end{gathered}
$$

Особое внимание к задаче Дирихле для уравнений смешанного типа стало проявляться после опубликования работы Ф. И. Франкля [1], где впервые отмечено, что проблемы трансзвуковой газовой динамики сводятся к этой задаче. А. В. Бицадзе [2] показал некорректность задачи Дирихле для уравнения Лаврентьева $u_{x x}+\operatorname{sgn} y u_{y y}=0$. После этой работы возникла проблема поиска смешанных областей, для которых задача Дирихле является корректно поставленной. В дальнейшем задача Дирихле для уравнений смешанного типа изучалась многими авторами [3-11]. Достаточно полную библиографию работ, посвященных этой тематике, можно найти в монографии [11]. Задача Дирихле для уравнений смешанного типа второго рода (1) при $k=0$ исследована в работах [11, с. 33-49], [12-18].

Данная работа является продолжением исследований автора [19,20], где первая граничная задача для уравнения (1) в прямоугольной области $D$ изучена при $0<k<1, m=0, a=0$ и $k \geqslant 1,0<m<1$ соответственно.

В данной работе установлен критерий единственности решения задачи Дирихле при $k<1$ и $1 \leqslant m<2$. Решение построено в виде суммы ряда Фурье-Бесселя. При обосновании равномерной сходимости построенного ряда возникает проблема малых знаменателей. Найдена оценка отделенности 
малого знаменателя от нуля с соответствующей асимптотикой, которая позволила обосновать сходимость построенного ряда в классе функций (2)-(4).

2. Критерий единственности решения задачи. Частные решения уравнения (1), не равные нулю на множестве $D^{+} \cup D^{-}$и удовлетворяющие нулевым граничным условиям (7) и (8), ищем в виде $u(x, y)=X(x) Y(y)$. Подставляя данное произведение в уравнение (1) относительно $X(x)$, получим следующую спектральную задачу:

$$
\begin{gathered}
X^{\prime \prime}(x)+\frac{k}{x} X^{\prime}(x)+\lambda^{2} X(x)=0, \quad 0<x<l, \\
X(0)=0, \quad X(l)=0,
\end{gathered}
$$

где $\lambda^{2}-$ постоянная разделения переменных.

Решение спектральной задачи (9) и (10) определяется формулой

$$
\tilde{X}_{n}(x)=x^{(1-k) / 2} J_{\frac{1-k}{2}}\left(\lambda_{n} x\right), \quad \lambda_{n}=\frac{\mu_{n}}{l}, \quad n=1,2,3, \ldots,
$$

где $J_{\nu}(\cdot)$ - функция Бесселя первого рода порядка $\nu, \mu_{n}-n$-ный корень уравнения

$$
J_{\frac{1-k}{2}}\left(\mu_{n}\right)=0 .
$$

Для удобства дальнейших вычислений данную систему функций ортонормируем:

$$
X_{n}(x)=\frac{1}{\left\|\tilde{X}_{n}\right\|_{L_{2, \rho}(0, l)}} \tilde{X}_{n}(x)
$$

где

$$
\begin{aligned}
\left\|\tilde{X}_{n}\right\|_{L_{2, \rho}(0, l)}=( & \left.\int_{0}^{l} \rho(x) \tilde{X}_{n}^{2}(x) d x\right)^{1 / 2}= \\
& =\left(\int_{0}^{l} x J_{\frac{1-k}{2}}^{2}\left(\frac{\mu_{n}}{l} x\right) d x\right)^{1 / 2}=\frac{l}{\sqrt{2}}\left|J_{\frac{3-k}{2}}\left(\mu_{n}\right)\right|, \quad \rho(x)=x^{k} .
\end{aligned}
$$

Известно [21, Sect. 18.1], что система собственных функций (11) ортогональна и полна в пространстве $L_{2}[0, l]$ с весом $x^{k}$.

Отметим, что для собственных значений спектральной задачи (9), (10) при больших $n$ справедлива следующая асимптотическая формула (по аналогии с [22, Sect. 6.5]):

$$
\mu_{n}=\lambda_{n} l=\pi n-\frac{k}{4} \pi+O\left(\frac{1}{n}\right) .
$$

Пусть $u(x, y)$ - решение задачи (2)-(8), удовлетворяющее условию

$$
\lim _{x \rightarrow l-0} X_{n}(x) u_{x}=0 .
$$

Следуя работам $[17,20]$, рассмотрим следующие функции:

$$
u_{n}(y)=\int_{0}^{l} u(x, y) x^{k} X_{n}(x) d x, \quad n=1,2,3, \ldots
$$


На основании (14) введем вспомогательные функции

$$
u_{n, \varepsilon}(y)=\int_{\varepsilon}^{l-\varepsilon} u(x, y) x^{k} X_{n}(x) d x
$$

где $\varepsilon>0$ - достаточно малое число. Дифференцируя равенство (15) по у дважды при $y \in(-\alpha, 0) \cup(0, \beta)$ и учитывая уравнение $(1)$, получим

$$
\begin{gathered}
u_{n, \varepsilon}^{\prime \prime}(y)=\int_{\varepsilon}^{l-\varepsilon} u_{y y}(x, y) x^{k} X_{n}(x) d x= \\
=(\operatorname{sgn} y)|y|^{-m} \int_{\varepsilon}^{l-\varepsilon}\left(a^{2} u-u_{x x}-\frac{k}{x} u_{x}\right) x^{k} X_{n}(x) d x= \\
=(\operatorname{sgn} y)|y|^{-m}\left(a^{2} u_{n, \varepsilon}(y)-\int_{\varepsilon}^{l-\varepsilon} \frac{\partial}{\partial x}\left(x^{k} u_{x}\right) X_{n}(x) d x\right)= \\
=(\operatorname{sgn} y)|y|^{-m}\left[a^{2} u_{n, \varepsilon}(y)-\left(\left.x^{k} u_{x} X_{n}(x)\right|_{\varepsilon} ^{l-\varepsilon}-\int_{\varepsilon}^{l-\varepsilon} x^{k} u_{x} X_{n}^{\prime}(x) d x\right)\right] .
\end{gathered}
$$

Из равенства (15) в силу уравнения (9) получим

$$
\begin{gathered}
u_{n, \varepsilon}(y)=-\frac{1}{\lambda_{n}^{2}} \int_{\varepsilon}^{l-\varepsilon} u(x, y) x^{k}\left(X_{n}^{\prime \prime}(x)+\frac{k}{x} X_{n}^{\prime}(x)\right) d x= \\
=-\frac{1}{\lambda_{n}^{2}} \int_{\varepsilon}^{l-\varepsilon} u(x, y) \frac{d}{d x}\left(x^{k} X_{n}^{\prime}(x)\right) d x= \\
=-\frac{1}{\lambda_{n}^{2}}\left(\left.x^{k} X_{n}^{\prime}(x) u\right|_{\varepsilon} ^{l-\varepsilon}-\int_{\varepsilon}^{l-\varepsilon} u_{x} x^{k} X_{n}^{\prime}(x) d x\right) .
\end{gathered}
$$

Из равенства (17) имеем

$$
\int_{\varepsilon}^{l-\varepsilon} u_{x} x^{k} X_{n}^{\prime}(x) d x=\lambda_{n}^{2} u_{n, \varepsilon}(y)+\left.x^{k} X_{n}^{\prime}(x) u\right|_{\varepsilon} ^{l-\varepsilon}
$$

Подставляя (18) в (16), получим

$$
\begin{aligned}
u_{n, \varepsilon}^{\prime \prime}(y)=(\operatorname{sgn} y)|y|^{-m}\left(a^{2} u_{n, \varepsilon}(y)-\left.x^{k} u_{x} X_{n}(x)\right|_{\varepsilon} ^{l-\varepsilon}-\right. & \\
& \left.-\lambda_{n}^{2} u_{n, \varepsilon}(y)-\left.x^{k} X_{n}^{\prime}(x) u\right|_{\varepsilon} ^{l-\varepsilon}\right) .
\end{aligned}
$$

Предварительно заметим, что следующий предел существует и конечен:

$$
\lim _{x \rightarrow 0+0} x^{k} X_{n}^{\prime}(x)=\frac{\sqrt{2} \lambda_{n}}{l\left|J_{\frac{3-k}{2}}\left(\mu_{n}\right)\right|} \lim _{x \rightarrow 0+0} x^{(k+1) / 2} J_{-\frac{k+1}{2}}\left(\lambda_{n} x\right)=\frac{2^{(k+2) / 2} \lambda_{n}^{(1-k) / 2}}{l\left|J_{\frac{3-k}{2}}\left(\mu_{n}\right)\right| \Gamma\left(\frac{1-k}{2}\right)} .
$$

Переходя в (19) к пределу при $\varepsilon \rightarrow 0$, с учетом граничных условий (7), (8), (10) и (13) получим, что $u_{n}(y)$ удовлетворяет дифференциальному уравнению

$$
u_{n}^{\prime \prime}(y)-(\operatorname{sgn} y)|y|^{-m}\left(a^{2}+\lambda_{n}^{2}\right) u_{n}(y)=0, \quad y \in(-\alpha, 0) \cup(0, \beta),
$$


условиям склеивания

$$
\begin{gathered}
u_{n}(0-0)=u_{n}(0+0), \lim _{y \rightarrow 0+0} y^{m-1} u_{n}^{\prime}(y)=-\lim _{y \rightarrow 0-0}(-y)^{m-1} u_{n}^{\prime}(y), 1<m<2, \\
u_{n}(0-0)=u_{n}(0+0), \quad \lim _{y \rightarrow 0+0} \frac{u_{n}^{\prime}(y)}{\ln y}=-\lim _{y \rightarrow 0-0} \frac{u_{n}^{\prime}(y)}{\ln (-y)}, \quad m=1
\end{gathered}
$$

и граничным условиям

$$
\begin{aligned}
u_{n}(\beta) & =\int_{0}^{l} u(x, \beta) x^{k} X_{n}(x) d x=\int_{0}^{l} \varphi(x) x^{k} X_{n}(x) d x=\varphi_{n}, \\
u_{n}(-\alpha) & =\int_{0}^{l} u(x,-\alpha) x^{k} X_{n}(x) d x=\int_{0}^{l} \psi(x) x^{k} X_{n}(x) d x=\psi_{n} .
\end{aligned}
$$

Аналогично работам $[15,20]$ найдем общее решение дифференциального уравнения (20), удовлетворяющее условиям (21) и (22)

$$
u_{n}(y)= \begin{cases}a_{n} \sqrt{y} I_{\frac{1}{2 q}}\left(p_{n} y^{q}\right), & y>0, \\ b_{n} \sqrt{-y} J_{\frac{1}{2 q}}\left(p_{n}(-y)^{q}\right), & y<0,\end{cases}
$$

где $I_{\nu}(\cdot)$ - модифицированная функция Бесселя первого рода порядка $\nu$,

$$
q p_{n}=\sqrt{a^{2}+\lambda_{n}^{2}}, \quad q=\frac{2-m}{2} \in(0,1 / 2] .
$$

Далее, удовлетворяя функции (25) граничными условиями (23) и (24), получим равенства для определения неизвестных коэффициентов $a_{n}$ и $b_{n}$ :

$$
a_{n} I_{\frac{1}{2 q}}\left(p_{n} \beta^{q}\right)=\varphi_{n} \beta^{-1 / 2}, \quad b_{n} J_{\frac{1}{2 q}}\left(p_{n} \alpha^{q}\right)=\psi_{n} \alpha^{-1 / 2} .
$$

Единственное решение системы (26) существует и определяется по формуле

$$
u_{n}(y)= \begin{cases}\frac{\sqrt{y} \varphi_{n} I_{\frac{1}{2 q}}\left(p_{n} y^{q}\right)}{\sqrt{\beta} I_{\frac{1}{2 q}}\left(p_{n} \beta^{q}\right)}, & y>0, \\ \frac{\sqrt{-y} \psi_{n} J_{\frac{1}{2 q}}\left(p_{n}(-y)^{q}\right)}{\sqrt{\alpha} J_{\frac{1}{2 q}}\left(p_{n} \alpha^{q}\right)}, & y<0\end{cases}
$$

при условии

$$
E(n)=J_{\frac{1}{2 q}}\left(p_{n} \alpha^{q}\right) \neq 0, \quad n \in \mathbb{N} .
$$

Пусть теперь $\varphi(x) \equiv 0$ и $\psi(x) \equiv 0$. Тогда из равенств $(23),(24),(27)$ и (14) следует

$$
\int_{0}^{l} u(x, y) x^{k} X_{n}(x) d x=0, \quad n=1,2,3, \ldots
$$

Отсюда в силу полноты системы (11) в пространстве $L_{2}[0, l]$ с весом $x^{k}$ следует, что $u(x, y)=0$ для всех $x \in[0, l]$ и при любом $y \in[-\alpha, \beta]$. В силу (2) функция $u(x, y)$ непрерывна на $\bar{D}$, следовательно, $u(x, y) \equiv 0$ на $\bar{D}$. 
Пусть при некоторых $\alpha, q, a, k, l$ и $n=s \in \mathbb{N}$ нарушено условие (28), то есть $E(s)=0$. Тогда однородная задача $(2)-(8)$, где $\varphi(x)=\psi(x) \equiv 0$, имеет нетривиальное решение

$$
u_{s}(x, y)=u_{s}(y) X_{s}(x)
$$

где

$$
u_{s}(y)=\left\{\begin{array}{cc}
0, & y \geqslant 0 \\
J_{\frac{1}{2 q}}\left(p_{s}(-y)^{q}\right) \sqrt{-y}, & y<0
\end{array}\right.
$$

$X_{s}(x)$ находятся по формуле (11).

Действительно, функция (29) в силу условия

$$
J_{\frac{1}{2 q}}\left(p_{s} \alpha^{q}\right)=0
$$

удовлетворяет однородным граничным условиям (6), где $\varphi(x)=\psi(x) \equiv 0$. Нетрудно доказать, что функция (29) также удовлетворяет условиям (2)-(5) и $(7),(8)$.

Поскольку функция Бесселя $J_{\frac{1}{2 q}}\left(p_{n} \alpha^{q}\right)$ имеет счетное множество нулей относительно $\alpha_{q}=\alpha^{q} / q$ при фиксированном $n$, приходим к следующему утверждению.

Теорема 1. Если существует решение задачи (2)-(8), удовлетворяющее условию (13), то оно единственно только тогда, когда выполняются условия (28) при всех $n \in \mathbb{N}$.

3. Обоснование существования решения задачи. Для обоснования существования решения задачи Дирихле необходимо показать существование чисел $\alpha, q, a, k, l$, таких, что при достаточно больших $n$ выражение $E(n)$ отделено от нуля с соответствующей асимптотикой.

ЛЕмма 1. Если

$$
\widetilde{\alpha_{q}}=\frac{\alpha^{q}}{q l}=\frac{p}{t}, \quad p, t \in \mathbb{N}, \quad(p, t)=1
$$

и выполнено условие

$$
k \neq \frac{1}{p}\left(4 r+t \frac{q-1}{q}-4 t d\right), \quad d \in \mathbb{Z}, \quad r \in \mathbb{N}_{0} \cap[0, t-1],
$$

то существуют положительные постоянные $C_{0} u n_{0}, n_{0} \in \mathbb{N}$, такие, что при всех $n>n_{0}$ справедлива оченка

$$
|\sqrt{n} E(n)| \geqslant C_{0}>0
$$

Дока за тельств о. На основании асимптотической формулы [23, Еq. $7.13(3)]$ для функции

$$
J_{\nu}(z)=\sqrt{\frac{2}{\pi z}} \cos \left(z-\frac{\pi}{2} \nu-\frac{\pi}{4}\right)+O\left(\frac{1}{z^{5 / 2}}\right), \quad z \rightarrow \infty,
$$


при достаточно больших $n>n_{1}$ имеем

$$
\begin{aligned}
J_{\frac{1}{2 q}}\left(p_{n} \alpha^{q}\right)=J_{\frac{1}{2 q}}\left(\widetilde{\alpha_{q}} \mu_{n} \widetilde{p_{n}}\right) & = \\
& =\sqrt{\frac{2}{\pi \widetilde{\alpha_{q}} \mu_{n} \widetilde{p_{n}}}} \sin \left(\widetilde{\alpha_{q}} \mu_{n} \widetilde{p_{n}}+\pi \frac{q-1}{4 q}\right)+O\left(n^{-5 / 2}\right),
\end{aligned}
$$

где

$$
p_{n} \alpha^{q}=\frac{\alpha^{q}}{q} \sqrt{a^{2}+\lambda_{n}^{2}}=\frac{\alpha^{q}}{q} \sqrt{a^{2}+\left(\frac{\mu_{n}}{l}\right)^{2}}=\frac{\alpha^{q}}{q l} \mu_{n} \sqrt{1+\left(\frac{a l}{\mu_{n}}\right)^{2}}=\widetilde{\alpha_{q}} \mu_{n} \widetilde{p_{n}}
$$

При этом натуральное число $n_{1}$ выбирается настолько большим, что при всех $n>n_{1}$ выполняется равенство (31) и при любых фиксированных $a \geqslant 0$ имеет место неравенство

$$
0 \leqslant \frac{a l}{\mu_{n}}<1
$$

Тогда

$$
\widetilde{p_{n}}=\left(1+\left(\frac{a l}{\mu_{n}}\right)^{2}\right)^{1 / 2}=1+\frac{1}{2}\left(\frac{a l}{\mu_{n}}\right)^{2}-\frac{1}{8}\left(\frac{a l}{\mu_{n}}\right)^{4}+\cdots=1+\theta_{n},
$$

где для $\theta_{n}$ справедлива оценка

$$
0 \leqslant \theta_{n}<\frac{1}{2}\left(\frac{a l}{\mu_{n}}\right)^{2}
$$

Отсюда на основании формулы (12)

$$
\mu_{n} \widetilde{p_{n}} \widetilde{\alpha_{q}}=\pi n \widetilde{\alpha_{q}}\left(1+\theta_{n}\right)-\frac{\pi}{4} k \widetilde{\alpha_{q}}\left(1+\theta_{n}\right)+O\left(\frac{1}{n}\right) .
$$

Тогда из соотношения (31) с учетом (33) получим

$$
\begin{aligned}
& \sqrt{n} J_{\frac{1}{2 q}}\left(p_{n} \alpha^{q}\right)=\sqrt{n} J_{\frac{1}{2 q}}\left(\mu_{n} \widetilde{p_{n}} \widetilde{\alpha_{q}}\right)= \\
&=B_{n} \sin \left[\pi n \tilde{\alpha_{q}}\left(1+\theta_{n}\right)-\frac{\pi}{4} k \tilde{\alpha_{q}}\left(1+\theta_{n}\right)+O\left(\frac{1}{n}\right)+\pi \frac{q-1}{4 q}\right]+ \\
&+O\left(\frac{1}{n^{2}}\right)=B_{1 n}+B_{2 n}
\end{aligned}
$$

где

$$
B_{n}=\frac{2}{\pi \sqrt{\widetilde{\alpha_{q}}\left(1+\theta_{n}-\frac{k}{4 n}\left(1+\theta_{n}\right)\right)+O\left(n^{-2}\right)}} .
$$

Поскольку последовательность $B_{n}$ имеет конечный положительный предел $B_{\infty}$, она ограничена и отделена от нуля.

Пусть теперь $\widetilde{\alpha_{q}}=p / t-$ рациональное число, $p, t \in \mathbb{N},(p, t)=1$. Разделим np на $t$ с остатком:

$$
n p=s t+r, \quad r, s \in \mathbb{N}_{0}, \quad 0 \leqslant r \leqslant t-1 .
$$


В силу оценки (32) существует конечный предел

$$
\lim _{n \rightarrow \infty}\left|B_{1 n}\right|=B_{\infty}\left|\sin \left[\frac{\pi r}{t}-\frac{\pi}{4} k \frac{p}{t}+\pi \frac{q-1}{4 q}\right]\right|=A_{1} \geqslant 0 .
$$

Тогда существует натуральное число $n_{2}$ такое, что при всех $n>n_{2}$

$$
\left|B_{1 n}\right| \geqslant \frac{1}{2} A_{1} \geqslant 0
$$

Теперь потребуем, чтобы постоянная $A_{1}$ была больше нуля, а это возможно только тогда, когда

$$
\frac{\pi r}{t}-\frac{\pi}{4} k \frac{p}{t}+\pi \frac{q-1}{4 q} \neq d \pi
$$

или

$$
\frac{r}{t}-\frac{k}{4} \frac{p}{t}+\frac{q-1}{4 q} \neq d
$$

при всех $d \in \mathbb{Z}$ и $r \in \mathbb{N}_{0} \cap[0, t-1]$. Из (34) имеем

$$
k \neq \frac{1}{p}\left(4 r+t \frac{q-1}{q}-4 t d\right) .
$$

Из неравенства (35) видно, что если $k$ принимает иррациональные значения, а $q$ рациональные значения и наоборот, то неравенство (34) всегда выполнено. При рациональных $k$ и $q$ условие (34) может нарушаться, поэтому потребуем, чтобы постоянные $k, q$ и $\widetilde{\alpha_{q}}$ удовлетворяли условию (35).

Таким образом, при выполнении условия (35) при всех $n \geqslant \max \left\{n_{1}, n_{2}\right\}$

$$
\left|B_{1 n}\right| \geqslant \frac{A_{1}}{2}>0
$$

Тем самым показана отделимость от нуля выражения $\sqrt{n} E(n)$ при больших $n$.

Если $E(n) \neq 0$ при $n=\overline{1, n_{0}}$ для указанных $\widetilde{\alpha_{q}}$ из леммы 1 и выполнена оценка (30) при $n>n_{0}$, то решение задачи (2)-(8) на основании частных решений (11) и (27) можно представить в виде суммы ряда Фурье-Бесселя:

$$
u(x, y)=\sum_{n=1}^{+\infty} u_{n}(y) X_{n}(x)
$$

Теперь покажем, что при определенных условиях относительно функций $\psi(x)$ и $\varphi(x)$ (см. (6)) ряд (36) сходится равномерно на замкнутой области $\bar{D}$, в которой его можно почленно дифференцировать по $x$ и $y$ дважды.

Рассмотрим следующие отношения:

$$
V_{n}(y)=\sqrt{\frac{y}{\beta}} \frac{I_{\frac{1}{2 q}}\left(p_{n} y^{q}\right)}{I_{\frac{1}{2 q}}\left(p_{n} \beta^{q}\right)}, \quad y \in[0, \beta]
$$




$$
W_{n}(y)=\sqrt{\frac{-y}{\alpha}} \frac{J_{\frac{1}{2 q}}\left(p_{n}(-y)^{q}\right)}{J_{\frac{1}{2 q}}\left(p_{n} \alpha^{q}\right)}, \quad y \in[-\alpha, 0] .
$$

Лемма 2. Для (37), (38) при достаточно больших п справедливы следующие оценки:

$$
\begin{gathered}
\left|V_{n}(y)\right| \leqslant 1, \quad y \in[0, \beta] \\
\left|W_{n}(y)\right| \leqslant C_{1}, \quad y \in[-\alpha, 0] \\
\left|V_{n}^{\prime}(y)\right| \leqslant C_{2} n, \quad\left|V_{n}^{\prime \prime}(y)\right| \leqslant C_{3} n^{2}, \quad \varepsilon \leqslant y \leqslant \beta ; \\
\left|W_{n}^{\prime}(y)\right| \leqslant C_{4} n, \quad\left|W_{n}^{\prime \prime}(y)\right| \leqslant C_{5} n^{2}, \quad-\alpha \leqslant y \leqslant-\varepsilon,
\end{gathered}
$$

где $C_{i}$-здесь и далее положстельные постоянные, $\varepsilon>0$-достаточное малое число.

Лемма 3. Если выполнена оценка (30) при $n>n_{0}$, то для таких $n$ справедливы следующие оченки:

$$
\begin{gathered}
\left|u_{n}(y)\right| \leqslant C_{6}\left(\left|\varphi_{n}\right|+\left|\psi_{n}\right|\right), \quad y \in[-\alpha, \beta] \\
\left|u_{n}^{\prime}(y)\right| \leqslant C_{7} n\left(\left|\varphi_{n}\right|+\left|\psi_{n}\right|\right), \quad\left|u_{n}^{\prime \prime}(y)\right| \leqslant C_{8} n^{2}\left(\left|\varphi_{n}\right|+\left|\psi_{n}\right|\right), \quad y \in[-\alpha,-\varepsilon] \cup[\varepsilon, \beta] .
\end{gathered}
$$

Доказательств о следует из леммы 2.

Лемма 4. При достаточно больших $n$ и при всех $x \in[\delta, l]$, где $\delta-$ достаточно малое число, справедливы следующие оченки:

$$
\left|X_{n}(x)\right| \leqslant C_{9}, \quad\left|X_{n}^{\prime}(x)\right| \leqslant C_{10} n, \quad\left|X_{n}^{\prime \prime}(x)\right| \leqslant C_{11} n^{2} .
$$

где $C_{i}$ - положстельные постоянные.

Доказ атель ст в о проводится аналогично работам [20,24].

Лемма 5. Если $\varphi(x) \in C^{4}[0, l] u \psi(x) \in C^{4}[0, l]$ и выполняются условия

$$
\begin{gathered}
\varphi(0)=\psi(0)=\varphi^{\prime}(0)=\psi^{\prime}(0)=\varphi^{\prime \prime}(0)=\psi^{\prime \prime}(0)=0, \\
\varphi(l)=\psi(l)=\varphi^{\prime}(l)=\psi^{\prime}(l)=\varphi^{\prime \prime}(l)=\psi^{\prime \prime}(l)=0,
\end{gathered}
$$

то справедливы оченки

$$
\left|\varphi_{n}\right| \leqslant \frac{C_{12}}{n^{4}}, \quad\left|\psi_{n}\right| \leqslant \frac{C_{13}}{n^{4}} .
$$

Дока з атель ст в о проводится аналогично работам [20,24].

Формально из ряда (36) почленным дифференцированием составим ряды

$$
u_{y}(x, y)=\sum_{n=1}^{+\infty} u_{n}^{\prime}(y) X_{n}(x), \quad u_{x}(x, y)=\sum_{n=1}^{+\infty} u_{n}(y) X_{n}^{\prime}(x) .
$$




$$
u_{y y}(x, y)=\sum_{n=1}^{+\infty} u_{n}^{\prime \prime}(y) X_{n}(x), \quad u_{x x}(x, y)=\sum_{n=1}^{+\infty} u_{n}(y) X_{n}^{\prime \prime}(x)
$$

Тогда на основании лемм 3 и 4 ряд (36) при любом $(x, y) \in \overline{D_{\delta}}$ мажорируется рядом

$$
C_{14} \sum_{n=n_{0}}^{+\infty}\left(\left|\varphi_{n}\right|+\left|\psi_{n}\right|\right)
$$

а ряды $(39)$ и $(40)$ при любом $(x, y) \in \overline{D_{\delta, \varepsilon}^{+}} \cup \overline{D_{\delta, \varepsilon}^{-}}-$соответственно рядами

$$
C_{15} \sum_{n=n_{0}}^{+\infty} n\left(\left|\varphi_{n}\right|+\left|\psi_{n}\right|\right), \quad C_{16} \sum_{n=n_{0}}^{+\infty} n^{2}\left(\left|\varphi_{n}\right|+\left|\psi_{n}\right|\right)
$$

где $D_{\delta, \varepsilon}^{+}=D^{+} \cap\{x>\delta\} \cap\{y>\varepsilon>0\}, D_{\varepsilon}^{-}=D^{-} \cap\{x<\delta\} \cap\{y<-\varepsilon<0\}$.

Согласно лемме 5 ряды (41) и (42) оцениваются соответственно рядами

$$
C_{17} \sum_{n=n_{0}}^{+\infty} n^{-4}, \quad C_{18} \sum_{n=n_{0}}^{+\infty} n^{-3}, \quad C_{19} \sum_{n=n_{0}}^{+\infty} n^{-2}
$$

Поскольку числовые ряды из (43) сходятся, на основании признака Вейерштрасса ряд (36) и ряды (39) и (40) сходятся равномерно соответственно на $\bar{D}, \overline{D_{\delta, \varepsilon}^{+}}$и $\overline{D_{\delta, \varepsilon}^{-}}$. Поэтому функция $u(x, y)$, определяемая равенством $(36)$, удовлетворяет условиям (2)-(8).

Если для указанных в лемме 1 чисел $\widetilde{\alpha_{q}}$ при некоторых $n=s=r_{1}, r_{2}, \ldots, r_{h}$, где $1 \leqslant r_{1}<r_{2}<\cdots<r_{h} \leqslant n_{0}, r_{i}, i=\overline{1, h}$, и $h$ - заданные натуральные числа, то $E(s)=0$. Тогда для разрешимости задачи (2)-(8) необходимо и достаточно, чтобы выполнялись условия

$$
\psi_{s}=0, \quad s=r_{1}, r_{2}, \ldots r_{h}
$$

В этом случае решение задачи (2)-(8) определяется в виде суммы ряда

$$
u(x, y)=\left(\sum_{n=1}^{r_{1}-1}+\cdots+\sum_{n=r_{h-1}+1}^{r_{h}-1}+\sum_{n=r_{h}+1}^{+\infty}\right) u_{n}(y) X_{n}(x)+\sum_{s} u_{s}(x, y),
$$

здесь в последней сумме $s$ принимает значения $r_{1}, r_{2}, \ldots, r_{h}$, функция $u_{s}(x, y)$ определяются по следующей формуле:

$$
u_{s}(x, y)=\left\{\begin{array}{cc}
\frac{\varphi_{s} \sqrt{y} I_{\frac{1}{2 q}}\left(p_{s} y^{q}\right)}{\sqrt{\beta} I_{\frac{1}{2 q}}\left(p_{s} \beta^{q}\right)} X_{s}(x), & y \geqslant 0, \\
C_{s} J_{\frac{1}{2 q}}\left(p_{s}(-y)^{q}\right) \sqrt{-y} X_{s}(x), & y<0,
\end{array}\right.
$$

где $C_{s}$ - произвольные постоянные, конечные суммы в (45) следует считать равными нулю, если нижний предел больше верхнего. 
Нетрудно проверить, что найденные решения (36) и (45) удовлетворяют условиям (13).

Таким образом, доказана следующая

Теорема 2. Пусть $\varphi(x), \psi(x) \in C^{4}[0, l]$ и выполнены условия

$$
\begin{gathered}
\varphi^{\prime}(0)=\psi^{\prime}(0)=\varphi^{\prime \prime}(0)=\psi^{\prime \prime}(0)=0, \\
\varphi(l)=\psi(l)=\varphi^{\prime \prime}(l)=\psi^{\prime \prime}(l)=0,
\end{gathered}
$$

а такэсе условия (30) при $n>n_{0}$. Тогда если $E(n) \neq 0$ nри $n=\overline{1, n_{0}}$, то задача (2)-(8) однозначно разрешима и это решение определяется рядом (36). Если же $E(n)=0$ при некоторых $n=s=r_{1}, r_{2}, \ldots, r_{h} \leqslant n_{0}$, то задача (2)-(8) разрешима только тогда, когда выполняются условия (44) и решение в этом случае определяется рядом (45).

Конкурирующие интересы. Я заявляю, что не имею конкурирующих интересов.

Авторская ответственность. Я несу полную ответственность за предоставление окончательной версии рукописи в печать. Окончательная версия рукописи мною одобрена.

Финансирование. Работа выполнена при поддержке Российского фонда фундаментальных исследований (проект № 16-31-50008-мол_нр).

\section{Библиографический список}

1. Франкль Ф. И. О задачах С. А. Чаплыгина для смешанных до- и сверхзвуковых течений // Изв. АН СССР. Сер. матем., 1945. Т. 9, № 2. С. 121-143.

2. Бицадзе А. В. Некорректность задачи Дирихле для уравнений смешанного типа // ДАН ССCP, 1953. Т. 122 , № 2. С. 167-170.

3. Шабат Б. В. Примеры решения задачи Дирихле для уравнения смешанного типа // ДАН СССР, 1957. Т. 112, № 3. С. 386-389.

4. Вахания Н. Н. Об одной особой задаче для уравнения смешанного типа // Tp. AH Груз. CCP, 1963. T. 3. C. 69-80.

5. Cannon J. R. A Dirichlet problem for an equation of mixed type with a discontinuous coefficient // Annali di Matematica, 1963. vol.61, no. 1. pp. 371-377. doi: 10.1007/BF02410656.

6. Нахушев А. М. Критерий единственности задачи Дирихле для уравнения смешанного типа в цилиндрической области // Дифферени. уравнения, 1970. Т. 6, № 1. С. 190-191.

7. Хачев М. М. Задача Дирихле для уравнения Трикоми в прямоугольнике // Дифферени. уравнения, 1975. Т. 11, №1. С. 151-160.

8. Хачев М. М. paper О задаче Дирихле для одного уравнения смешанного типа // Дифферени. уравнения, 1976. Т. 12, №1. С. 137-143.

9. Солдатов А. П. Задача типа Дирихле для уравнения Лаврентьева-Бицадзе. I. Теоремы единственности // Докл. РАН, 1993. Т. 332, №6. С. 696-698.

10. Солдатов А. П. Задача типа Дирихле для уравнения Лаврентьева-Бицадзе. II. Теоремы существования // Докл. РАН, 1993. Т. 333, № 1. С. 16-18.

11. Хачев М. М. Первая краевая задача для линейных уравнений смешанного типа. Нальчик: Эльбрус, 1998. 168 с.

12. Сохадзе Р. С. Первая краевая задача для уравнения смешанного типа с весовыми условиями склеивания вдоль линии параболического вырождения // Дифференц. уравнения, 1981. Т. 17, №1. С. 150-156.

13. Сохадзе Р. С. О первой краевой задаче для уравнения смешанного типа в прямоугольнике // Дифференц. уравнения, 1983. Т. 19, № 1. С. 127-134.

14. Сабитов К. Б. Задача Дирихле для уравнений смешанного типа в прямоугольной области // Докл. РАН, 2007. Т. 413, №1. С. 23-26. 
15. Сабитов К. Б., Сулейманова А. Х. Задача Дирихле для уравнения смешанного типа второго рода в прямоугольной области // Изв. вузов. Матем., 2007. № 4. С. 45-53.

16. Сабитов К. Б., Сулейманова А. Х. Задача Дирихле для уравнения смешанного типа с характеристическим вырождением в прямоугольной области // Изв. вузов. Матем., 2009. № 11. C. $43-52$.

17. Сабитов К. Б., Вагапова Э. В. Задача Дирихле для уравнения смешанного типа с двумя линиями вырождения в прямоугольной области // Дифферени. уравнения, 2013. Т. 49, № 1. С. $68-78$.

18. Хайруллин Р. С. К задаче Дирихле для уравнения смешанного типа второго рода с сильным вырождением // Дифферени. уравнения, 2013. Т. 49, № 4. С. 528-534. doi : 10 . 1134/S0374064113040122.

19. Сафина Р. М. Задача Дирихле для уравнения Пулькина в прямоугольной области // Вестн. СамГУ. Естественнонаучн. сер., 2014. № 10(121). С. 91-101.

20. Сафина Р. М. Задача Келдыша для уравнения смешанного типа второго рода с оператором Бесселя // Дифферени. уравнения, 2015. Т. 51, №10. С. 1354-1366. doi: 10.1134/ S0374064115100106.

21. Watson G. N. A Treatise on the Theory of Bessel Functions. Cambridge: Cambridge University Press, 1944. viii +804 pp.

22. Olver F. W. J. Differential equations with irregular singularities; Bessel and confluent hypergeometric functions (Chapter 7) / Asymptotics and Special Functions. Boston: Academic Press, Inc., 1974. pp. 229-278. doi: 10.1016/B978-0-12-525850-0.50012-2.

23. Erdélyi A., Magnus W., Oberhettinger F., Tricomi F. G. Higher transcendental functions. vol. II / Bateman Manuscript Project. New York, Toronto, London: McGraw-Hill Book Co., Inc., 1953. xvii +396 pp.

24. Сафина Р. М. Задача Келдыша для уравнения Пулькина в прямоугольной области // Вестн. СамГУ. Естественнонаучн. сер., 2015. №3(125). С. 53-63. 
MSC: 35M10, 35M12

\title{
The Dirichlet problem for a mixed-type equation with strong characteristic degeneracy and a singular coefficient
}

\section{R. M. Safina}

Volga Region State Academy of Physical Culture, Sport and Tourism, 35, Universiade Village, Kazan, 420010, Russian Federation.

\begin{abstract}
In this paper we consider the first boundary value problem in a rectangular area for a mixed-type equation of the second kind with a singular coefficient. The criterion of the uniqueness of the problem solution is determined. The uniqueness of the problem solution is proved on the basis of completeness of the system of eigenfunctions of the corresponding onedimensional spectral problem. The solution of the problem is built explicitly as a sum of Fourier-Bessel. There is the problem of the small denominators that appears when justifying the uniform convergence of the constructed series. In this regard, an evaluation of separateness from zero with a corresponding small denominator asymptotic behavior is found. This estimate has allowed to prove the convergence of the series and its derivatives up to the second order, and the existence theorem for the class of regular solutions of this equation.
\end{abstract}

Keywords: mixed-type equation, Dirichlet problem, singular coefficient, spectral method, uniqueness, Fourier-Bessel series, small denominators, existence.

Received: $3^{\text {rd }}$ June, $2016 /$ Revised: $17^{\text {th }}$ January, $2017 /$

Accepted: $13^{\text {th }}$ March, $2017 /$ First online: $27^{\text {th }}$ April, 2017

Competing interests. I declare I have no competing interests.

Author's Responsibilities. I take full responsibility for submitting the final manuscript in print. I approved the final version of the manuscript.

Funding. This work was supported by the Russian Foundation for Basic Research (project no. 16-31-50008-mol_nr).

\section{References}

1. Frankl F. To the theory of the Laval nozzle, Izv. Akad. Nauk SSSR Ser. Mat., 1945, vol. 9, no. 2, pp. 121-143 (In Russian).

2. Bitsadze A. V. The incorrectness of the Dirichlet problem for equations of mixed type, Dokl. Akad. Nauk SSSR, 1953, vol.122, no. 2, pp. 167-170 (In Russian).

\section{Research Article}

(2) (1) The content is published under the terms of the Creative Commons Attribution 4.0 International License (http://creativecommons.org/licenses/by/4.0/)

Please cite this article in press as:

Safina R. M. The Dirichlet problem for a mixed-type equation with strong characteristic degeneracy and a singular coefficient, Vestn. Samar. Gos. Tekhn. Univ., Ser. Fiz.-Mat. Nauki [J. Samara State Tech. Univ., Ser. Phys. Math. Sci.], 2017, vol. 21, no. 1, pp. 80-93. doi: 10.14498/vsgtu1495 (In Russian).

\section{Author's Details:}

Rimma M. Safina (1) http://orcid.org/0000-0002-5787-1427

Senior Lecturer; Dept. of Physical and Mathematical Disciplines and Information Technologies; e-mail: rimma77705@mail.ru 
3. Shabat B. V. Examples of solving the Dirichlet problem for equations of mixed type, Dokl. Akad. Nauk SSSR, 1957, vol.112, no. 3, pp. 386-389 (In Russian).

4. Vakhaniya N. N. On a particular problem for the mixed type, Trudy Akad. Nauk Gruz. SSR, 1963, vol. 3, pp. 69-80 (In Russian).

5. Cannon J. R. A Dirichlet problem for an equation of mixed type with a discontinuous coefficient, Annali di Matematica, 1963, vol.61, no. 1, pp. 371-377. doi: 10.1007/BF02410656.

6. Nakhushev A. M. A uniqueness condition of the Dirichlet problem for an equation of mixed type in a cylindrical domain, Differ. Uravn., 1970, vol. 6, no. 1, pp. 190-191 (In Russian).

7. Khachev M. M. The Dirichlet problem for the Tricomi equation in a rectangle, Differ. Uravn., 1975, vol.11, no. 1, pp. 151-160 (In Russian).

8. Khachev M. M. The Dirichlet problem for a certain equation of mixed type, Differ. Uravn., 1976, vol. 12, no. 1, pp. 137-143 (In Russian).

9. Soldatov A. P. Dirichlet-type problems for the Lavrent'ev-Bitsadze equation. I. Uniqueness theorems, Dokl. Math., 1994, vol.48, no. 2, pp. 410-414.

10. Soldatov A. N. Dirichlet-type problems for the Lavrent'ev-Bitsadze equation. II. Existence theorems, Dokl. Math., 1994, vol.48, no. 3, pp. 16-18.

11. Khachev M. M. Pervaia kraevaia zadacha dlia lineinykh uravnenii smeshannogo tipa [The First Boundary Value Problem for Linear Equations of Mixed Type]. Nalchik, Elbrus, 1998, 168 pp. (In Russian)

12. Sokhadze R. I. The first boundary value problem for an equation of mixed type with weighted glueing conditions along a line of parabolic degeneration, Differ. Uravn., 1981, vol. 17, no. 1, pp. 150-156 (In Russian).

13. Sokhadze R. I. The first boundary value problem for an equation of mixed type in a rectangle, Differ. Uravn., 1983, vol. 19, no. 1, pp. 127-134 (In Russian).

14. Sabitov K. B. The Dirichlet problem for equations of mixed type in a rectangular domain, Dokl. Math., 2007, vol. 75, no. 2, pp. 193-196.

15. Sabitov K. B., Suleimanova A. Kh. The Dirichlet problem for a mixed-type equation of the second kind in a rectangular domain, Russian Math. (Iz. VUZ), 2007, vol.51, no. 4, pp. 42-50. doi : 10.3103/S1066369X07040068.

16. Sabitov K. B., Suleimanova A. Kh. The Dirichlet problem for a mixed-type equation with characteristic degeneration in a rectangular domain, Russian Math. (Iz. VUZ), 2009, vol. 53, no. 11, pp. 37-45. doi : 10.3103/S1066369X0911005X.

17. Sabitov K. B., Vagapova E. V. Dirichlet problem for an equation of mixed type with two degeneration lines in a rectangular domain, Differ. Equ., 2013, vol.49, no. 1, pp. 68-78. doi: 10.1134/S0012266113010072.

18. Khairullin R. S. On the Dirichlet problem for a mixed-type equation of the second kind with strong degeneration, Differ. Equ., 2013, vol.49, no.4, pp. 510-516. doi:10.1134/ S0012266113040113.

19. Safina R. M. Dirichlet problem for Pulkin's equation in a rectangular domain, Vestnik SamGU. Estestvenno-Nauchnaya Ser., 2014, no.10(121), pp. 91-101 (In Russian).

20. Safina R. M. Keldysh problem for a mixed-type equation of the second kind with the Bessel operator, Differ. Equ., 2015, vol.51, no.10, pp. 1347-1359. doi: 10.1134/ S0012266115100109.

21. Watson G. N. A Treatise on the Theory of Bessel Functions. Cambridge, Cambridge University Press, 1944, viii +804 pp.

22. Olver F. W. J. Differential equations with irregular singularities; Bessel and confluent hypergeometric functions (Chapter 7), In: Asymptotics and Special Functions. Boston, Academic Press, Inc., 1974, pp. 229-278. doi: 10.1016/B978-0-12-525850-0.50012-2.

23. Erdélyi A., Magnus W., Oberhettinger F., Tricomi F. G. Higher transcendental functions, vol. II, Bateman Manuscript Project. New York, Toronto, London, McGraw-Hill Book Co., Inc., 1953, xvii +396 pp.

24. Safina R. M. Keldysh problem for Pulkin's equation in a rectangular domain, Vestnik SamGU. Estestvenno-Nauchnaya Ser., 2015, no.3(125), pp. 53-63 (In Russian). 